\title{
Modulating the stress response of $E$. coli at GaN interfaces using surface charge, surface chemistry, and genetic mutations
}

Sara Gleco, † Pramod Reddy, § Ronny Kirste, § Ramón Collazo, † Dennis LaJeunesse, ¥ Albena Ivanisevic †*

† Department of Materials Science and Engineering, North Carolina State University, Raleigh, North Carolina, 27695, USA

¥ Department of Nanoscience, Joint School of Nanoscience and Nanoengineering, University of North Carolina Greensboro

§Adroit Materials, 2054 Kildaire Farm Road, Suite 205, Cary, North Carolina 27518, United States

*author to whom correspondence should be addressed: ivanisevic@ncsu.edu

5 pages, 4 figures, 1 table 
Figure S1. Water contact angle

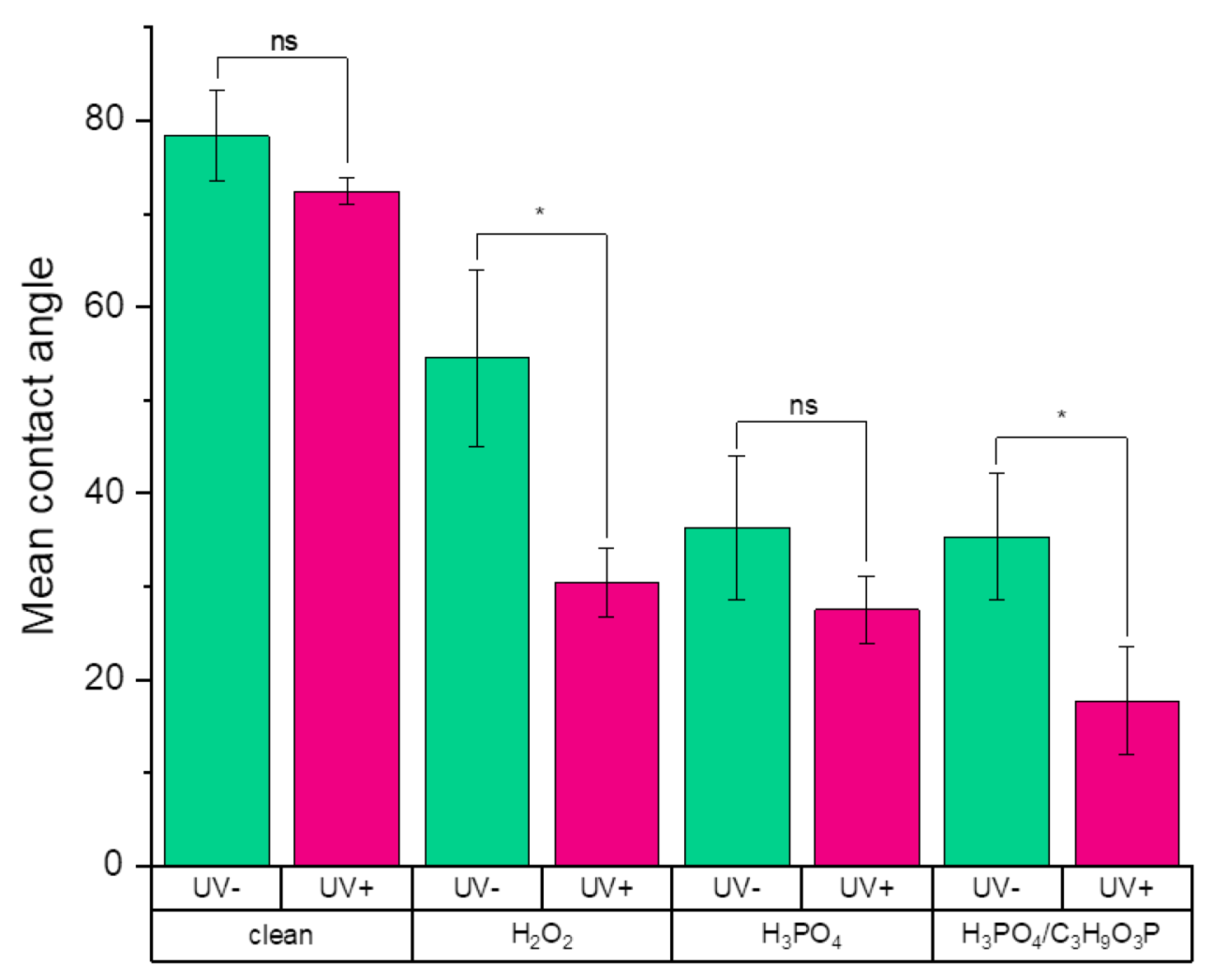


Figure S2. Glass coverslip surface potential

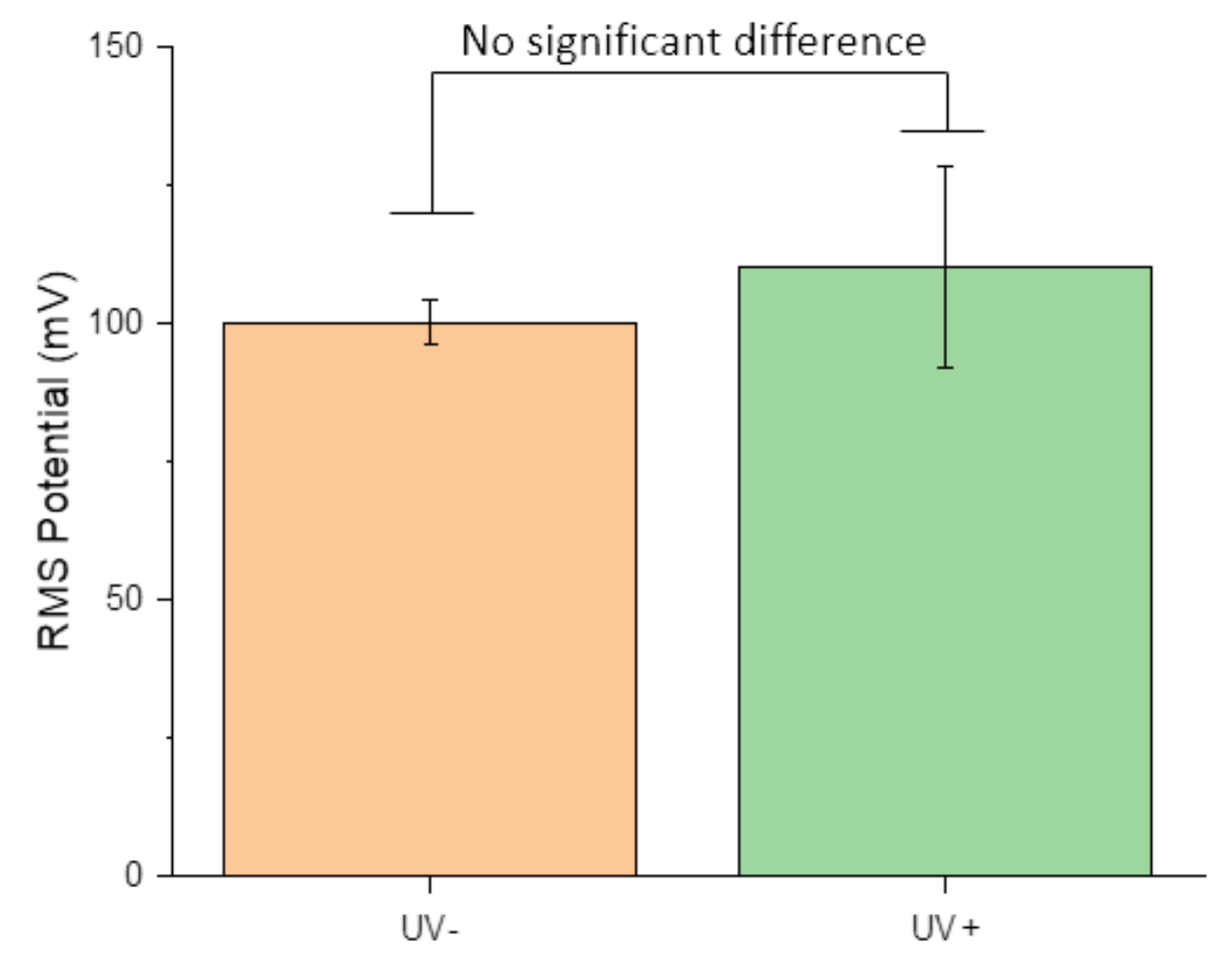


Table S1. Comparison of $\mathrm{ROS}$ and $\mathrm{Ca}^{2+}$ levels in bacteria exposed to different surface conditions. Complements Figs. S3-S8. Statistical difference indicated by $\uparrow$ (GaN greater than control), $\downarrow$ (GaN less than control), - (no difference between GaN and control).

\begin{tabular}{|c|c|c|c|c|c|c|c|c|c|}
\hline & \multicolumn{4}{|c|}{ UV+ vs. UV- } & & \multicolumn{2}{|c|}{ vs. Coverslip } & \multicolumn{2}{|c|}{ vs. $1 \mu \mathrm{M} \mathrm{H}_{2} \mathrm{O}_{2}$} \\
\hline UV & Sample & $\begin{array}{c}\text { Surface } \\
\text { charge }\end{array}$ & $\begin{array}{c}\text { Contact } \\
\text { Angle }\end{array}$ & Roughness & $\begin{array}{c}\text { E. coli } \\
\text { mutant }\end{array}$ & $\begin{array}{l}\text { ROS } \\
123 \\
\end{array}$ & $\begin{array}{l}\mathrm{Ca}^{2+} \\
123\end{array}$ & $\begin{array}{l}\text { ROS } \\
123\end{array}$ & $\begin{array}{l}\mathbf{C a}^{2+} \\
123\end{array}$ \\
\hline \multirow{12}{*}{$\sum_{3}^{+}$} & \multirow{3}{*}{ clean } & \multirow{3}{*}{$\uparrow$} & \multirow{3}{*}{-} & \multirow{3}{*}{-} & WT & $-\downarrow \downarrow$ & $\downarrow--$ & $\downarrow--$ & $\downarrow \downarrow-$ \\
\hline & & & & & csgG & $\downarrow--$ & $--\downarrow$ & --- & $\downarrow \downarrow \downarrow$ \\
\hline & & & & & flic & --- & $\downarrow-\uparrow$ & --- & $\downarrow-\uparrow$ \\
\hline & \multirow{3}{*}{$\mathrm{H}_{2} \mathrm{O}_{2}$} & \multirow{3}{*}{$\uparrow$} & \multirow{3}{*}{$\downarrow$} & \multirow{3}{*}{-} & WT & $\downarrow \downarrow \downarrow \downarrow$ & 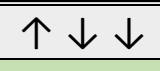 & $\downarrow--$ & $--\downarrow$ \\
\hline & & & & & $\operatorname{csgG}$ & --- & $\uparrow \uparrow \downarrow$ & --- & $--\downarrow$ \\
\hline & & & & & flic & $-\downarrow-$ & $-\uparrow \uparrow$ & $\downarrow--$ & $\downarrow \uparrow \uparrow$ \\
\hline & \multirow{3}{*}{$\mathrm{H}_{3} \mathrm{PO}_{4}$} & \multirow{3}{*}{-} & \multirow{3}{*}{-} & \multirow{3}{*}{-} & WT & $-\downarrow \downarrow$ & $\downarrow-\downarrow$ & $\downarrow--$ & $\downarrow \uparrow \downarrow$ \\
\hline & & & & & $\operatorname{csgG}$ & $\downarrow-\downarrow$ & $-\uparrow \downarrow$ & --- & $\downarrow-\downarrow$ \\
\hline & & & & & flic & --- & $-\downarrow \uparrow$ & $\downarrow--$ & $\downarrow \downarrow \downarrow$ \\
\hline & \multirow{3}{*}{$\mathrm{H}_{3} \mathrm{PO}_{4} / \mathrm{C}_{3} \mathrm{H}_{9} \mathrm{O}_{3} \mathrm{P}$} & \multirow{3}{*}{-} & \multirow{3}{*}{$\downarrow$} & \multirow{3}{*}{-} & WT & $-\downarrow \downarrow$ & $-1-\uparrow-$ & $\downarrow--$ & $\downarrow \downarrow$ \\
\hline & & & & & $\operatorname{csg} G$ & $\uparrow--$ & $-\downarrow \uparrow$ & --- & $-\uparrow \downarrow$ \\
\hline & & & & & flic & --- & $--\uparrow$ & --- & $--\uparrow$ \\
\hline \multirow{12}{*}{3} & \multirow{3}{*}{ clean } & \multirow{3}{*}{$\uparrow$} & \multirow{3}{*}{-} & \multirow{3}{*}{-} & WT & $-\downarrow-$ & $\downarrow--$ & $\downarrow \downarrow \uparrow$ & $\downarrow \uparrow-$ \\
\hline & & & & & $\operatorname{csgG}$ & --- & $--\downarrow$ & --- & $\downarrow \downarrow \downarrow$ \\
\hline & & & & & flic & $\uparrow \downarrow-$ & $--\uparrow$ & --- & $\downarrow \uparrow \uparrow$ \\
\hline & \multirow{3}{*}{$\mathrm{H}_{2} \mathrm{O}_{2}$} & \multirow{3}{*}{$\uparrow$} & \multirow{3}{*}{$\downarrow$} & \multirow{3}{*}{-} & WT & $-\downarrow \downarrow$ & $-\downarrow \downarrow$ & $\downarrow \downarrow-$ & $--\downarrow$ \\
\hline & & & & & $\operatorname{csgG}$ & --- & $\uparrow-\downarrow$ & --- & $\uparrow-\downarrow$ \\
\hline & & & & & flic & $-\downarrow-$ & $-\uparrow \uparrow$ & $\downarrow \downarrow-$ & $\downarrow \uparrow-$ \\
\hline & \multirow{3}{*}{$\mathrm{H}_{3} \mathrm{PO}_{4}$} & \multirow{3}{*}{-} & & & WT & $-\downarrow \downarrow$ & --- & $\downarrow \downarrow-$ & $\downarrow--$ \\
\hline & & & - & - & $\operatorname{csg} G$ & --- & $--\downarrow$ & --- & $\downarrow-\downarrow$ \\
\hline & & & & & flic & $-\downarrow \uparrow$ & $-\downarrow \uparrow$ & $\downarrow-\uparrow$ & $\downarrow \downarrow \downarrow$ \\
\hline & & & & & WT & $-\downarrow \downarrow$ & $\downarrow--$ & $-\downarrow-$ & $\downarrow \uparrow-$ \\
\hline & $\mathrm{H}_{3} \mathrm{PO}_{4} / \mathrm{C}_{3} \mathrm{H}_{9} \mathrm{O}_{3} \mathrm{P}$ & - & $\downarrow$ & - & $\operatorname{csg} G$ & --- & $-\downarrow \uparrow$ & $\downarrow--$ & $-\uparrow \downarrow$ \\
\hline & & & & & flic & $--\uparrow$ & --- & $\downarrow--$ & --- \\
\hline
\end{tabular}


Figure S3. ROS levels including controls measured a) one, b) two, and c) three hours after initial surface exposure.

Significant differences indicated in Table S1.
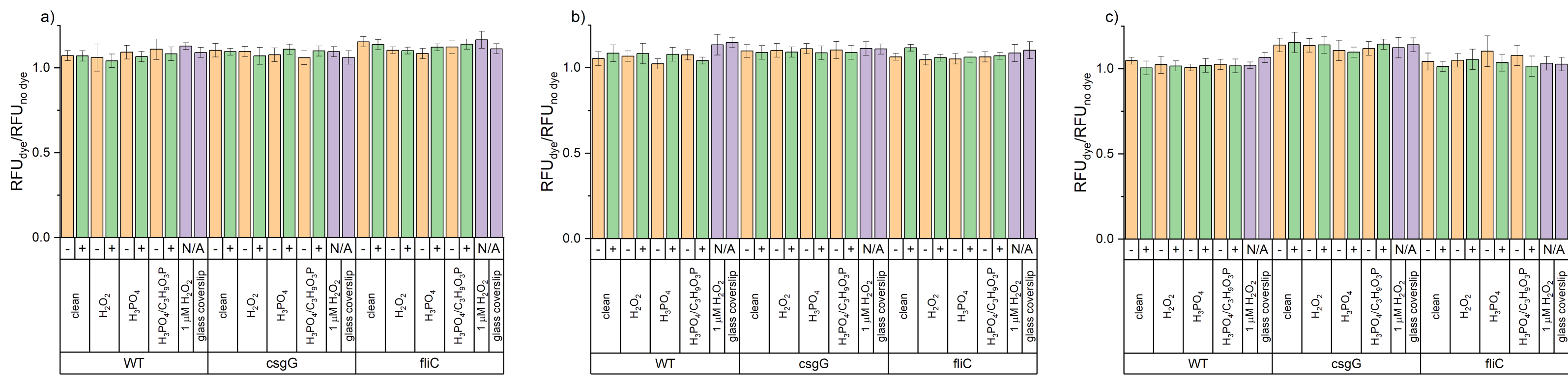
Figure S4. $\mathrm{Ca}^{2+}$ levels including controls measured a) one, b) two, and c) three hours after initial surface exposure Significant differences indicated in Table S1.
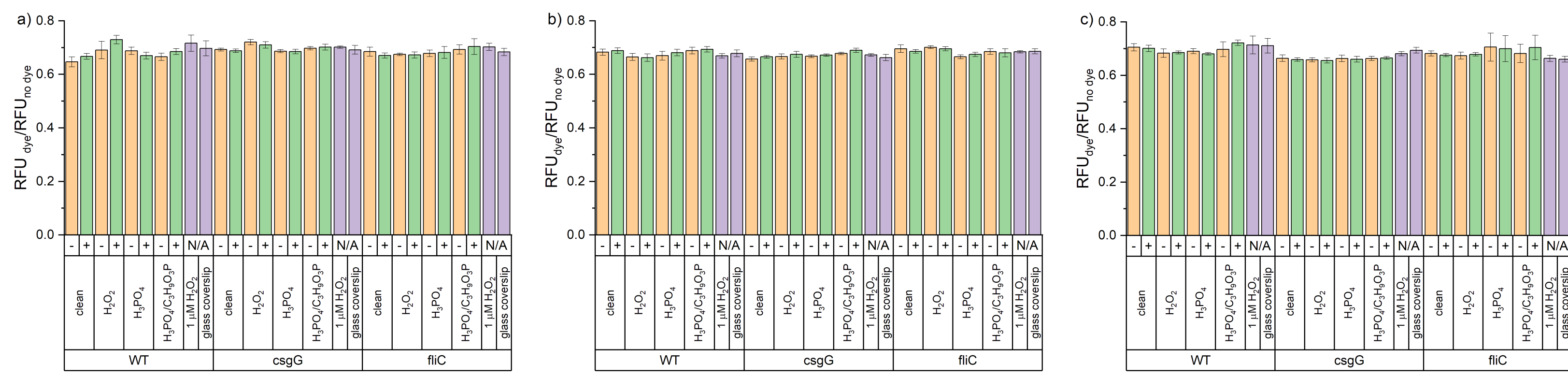